gen. Der Anreiz zu solchen öffentlich-privaten Partnerschaften ergibt sich nicht einseitig durch einen der beiden Partner, sondern gerade durch die spezielle Ausrichtung und Gestaltung einer langfristigen, fairen und vertraglich fixierten Partnerschaft beider, bei der neue und Erfolg versprechende Wege einer effizienteren Zusammenarbeit zwischen öffentlicher Hand und Privatsektor genutzt werden - in diesem Fall nicht nur zur Kosteneinsparung, sondern für etwas Besseres als den Freiheitsentzug.

\section{Literatur:}

Bertelsmann Stiftung u.a. (Hg.): Prozessleitfaden Public Private Partnership, URL: http://www.initiatived21.de/ themen/egovernment_pppleitfaden/doc/16_10577646 82.pdf vom 19.12.05

Cornel, Heinz: Gemeinnützige Arbeit zur Abwendung der Vollstreckung von Ersatzfreiheitsstrafen und als selb- ständige Sanktion, in: Prittwitz, Cornelius u. a. (Hg.): Festschrift für Klaus Lüderssen, Baden-Baden 2002, S. 821-834

Jehle, Jörg Martin; Feuerhelm, Wolfgang; Block, Petra: Gemeinnützige Arbeit statt Ersatz-freiheitsstrafe - Berichte, Materialien, Arbeitspapiere, Heft 4, Kriminologische Zentralstelle, Wiesbaden 1990

Kähler, Anja: Tilgung uneinbringlicher Geldstrafen durch gemeinnützige Arbeit. Praktische Möglichkeiten der Haftvermeidung - untersucht und erörtert am Beispiel des Praxisprojektes Gemeinnützige Arbeit beim Caritasverband Geldern-Kaevelaer e.V.. Münster: LIT 2003

Keicher, Rolf; Anhorn, Roland: Privatisierung als Chance? Straffälligenhilfe zwischen marktwirtschaftlicher und staatlicher Steuerung. Freiburg: Lambertus 2005

Kawamura, Gabriele: Gemeinnützige Arbeit in Bayern am Schnittpunkt von Sozialer Arbeit und Justiz. Fakten Erfahrungen - Lösungen. Nürnberg: Georg-SimonOhm-Fachhochschule 2000

Kawamura-Reindl, Gabriele; Reindl, Richard: Rahmenbedingungen erfolgreicher Ableistung gemeinnütziger Arbeit: Evaluationsergebnisse des Modells der Fachstel- len in Nordrhein-Westfalen, in Neue Kriminalpolitik, 15. Jg., H. 2/2003, S.49-52

Schneider, Ursula: Gemeinnützige Arbeit als Zwischensanktion, in Monatsschrift für Kriminologie und Strafrechtsreform, 84. Jg., H. 4/2001, S. 273-287

Sohn, Werner (1999). Gutachten der Kriminologischen Zentralstelle zur kurzen Freiheitsstrafe und zur Ersatzfreiheitsstrafe im Auftrag des Bundesministeriums der Justiz. Bearbeitungszeitraum: 1. Mai - 1. August 1999. Wiesbaden: KrimZ

Weiß, Manfred: Geleitwort des bayerischen Staatsministers der Justiz, in Kawamura 2000, S. 5

Gabriele Kawamura-Reindl ist Kriminologin und Sozialarbeiterin, Professorin am Fachbereich Sozialwesen der Georg-Simon-OhmFachhochschule Nürnberg und Mitherausgeberin der NK.

\title{
Auf dem Weg zu einem europäischen Modell von Bewährungshilfe?
}

m Frühjahr 2004 veröffentlichte das Justizministerium Baden-Württemberg in diversen Printmedien Europas eine »Beschränkte Ausschreibung mit öffentlichem Teilnahmewettbewerb «. Die Leistung, die von den Anbietern erwartet wurde, war nichts weniger als die Erledigung der Aufgaben der Bewährungs- und Gerichtshilfe in zwei Gerichtbezirken im Rahmen eines zeitlich auf zwei Jahre beschränkten Pilotverfahrens. Im Anschluss an diese Pilotphase ist die flächendeckende Aufgabenübertragung im gesamten Land BadenWürttemberg vorgesehen. Aufgabe des Anbieters war die Erstellung eines fachlichen und organisatorischen Konzepts für die Durchführung der Pilotierung, wobei größtmögliche Effizienz und Effektivität gewährleistet und nach einheitlichen und zweckmäßigen Standards sichergestellt sein soll. Weiter muss die Übertragbarkeit der organisatorischen und fachlichen Konzeption auf die übrigen Landgerichtsbezirke gesichert sein.

Diese Entscheidung löste heftige fachpolitische Kontroversen aus, deren Ton sich naturgemäß verschärfte, als im Oktober 2004 das Justizministerium den Zuschlag für diesen Auftrag nicht dem favorisierten eigens zu diesem Zweck von den Landesverbänden der Bewährungshilfe gegründeten lokalen Sozialunternehmen, sondern von sieben Bewerbern dem österreichischen Unternehmen »Neustart « erteilt wurde. Ausschlaggebend für diese Entscheidung war laut Pressemitteilungen u. a. dass »Neustart« als Unternehmen langjährige
Erfahrung mit der Durchführung von sozialarbeiterischen Leistungen für die Justiz aufweist, über eine übertragbare Organisationsstruktur sowie über ein Netz von ehrenamtlichen Bewährungshelfern verfügt und ein überzeugendes Projektkonzept vorlegte.

Nach der Zuschlagserteilung wurden zwischen dem Justizministerium und dem Sozialunternehmen »Neustart« weitere Details ausverhandelt und vertraglich festgelegt. Die einzelnen Ziele wurden in einem Projektplan festgelegt und das begleitende Projektcontrolling einem gemeinsam besetzten Lenkungsausschuss übertragen. Eine wissenschaftliche Evaluierung konnte wegen fehlender Finanzierbarkeit nicht vergeben werden. Seit 1.1.2005 wird das Projekt umgesetzt und verläuft laut Aussage des zuständigen Beamten des Justizministeriums Michael Steindorfer außerordentlich zufriedenstellend. U.a. wurden bereits 65 Personen gefunden, die bereit und fähig sind, als ehrenamtliche Bewährungshelfer selbständig und eigenverantwortlich Betreuungsarbeit zu leisten, womit in einem zentralen Bereich des Projekts die Zielgröße von 90 bis 110 nahezu erreicht wurde. Die Wogen haben sich weitgehend geglättet, was auch auf die gelungene Informationspolitik des Sozialunternehmens in den Medien und innerhalb der Fachöffentlichkeit zurückzuführen ist. Dementsprechend glücklich ist man auch in der Wiener Zentrale von »Neustart « und bereitet sich laut Auskunft des für die organisatorische
Durchführung verantwortlichen Geschäftführers Wolfgang Herrmann bereits auf den nach EURichtlinien vorgesehenen »Wettbewerblichen Dia-

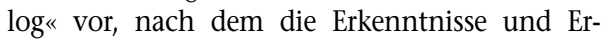
gebnisse des Pilotversuchs in allen Gerichtsbezirken Baden-Württembergs implementiert werden sollen. Diese Entwicklung entspricht dem Leitbild von »Neustart", der sich auch als europaweit tätige Expertenorganisation in Sachen Bewährungshilfe und anderen persönliche Dienstleistungen in der Justiz etablieren will.

Nach den mir vorliegenden Unterlagen handelt es sich hier um ein sehr gelungenes Beispiel einer Privatisierung und man kann den Projektverantwortlichen dazu nur gratulieren. Ich halte das Projekt für einen wichtigen Beitrag europäischer Integrationsbemühungen im Bereich des Sozialen, bei der die Qualität der Leistungserbringung für die KlientInnen im großen und ganzen verbessert werden konnte und weitere Qualitätsverbesserungen erwartet werden können. Das liegt auch in der humanistischen Tradition des Vereins »Neustart « und der dort entwickelten sozialarbeiterischen und organisatorischen Kompetenzen begründet. Es ist kein Unternehmen der "New Social Economy«, sondern ging in seiner Geschichte durch viele Krisen und weist auch Schwächen auf. Bevor ich das Projekt zur Nachahmung weiterempfehle, bedarf es jedoch einiger vertiefender Analysen und Überlegungen. 


\section{Europäische Entwicklungstrends bei der Modernisierung der Sozialen Dienste}

Nach einer Befragung der Beobachtungsstelle für die Entwicklung sozialer Dienste in Europa unter den 25 Mitgliedsstaaten im Jahre 2004 wurden von diesen übereinstimmende Entwicklungstrends herausgearbeitet (Newsletter vom 6.10.2005 www.eupik.org):

- Es werden Aufgaben des öffentlichen Sektors von den staatlichen Verwaltungen in Richtung privater Anbieter verlagert werden, was eine Änderung der Rolle des Staates nach sich ziehen wird;

- Effizienz- und Ergebnisorientierung werden gestärkt werden;

- Benchmarking und Qualitätsmanagment werden eingeführt werden;

- Organisationen werden ihre Strukturen dezentralisieren, um die Dienste auf lokaler und regionaler Ebene steuern zu können;

- Vernetzte und integrierte Versorgungsstrukturen werden aufgebaut werden;

- Es werden sich öffentliche und private Partnerschaften entwickeln;

- Leistungserbringung auf gesteuerten Märkten werden zunehmen;

- Elemente der Wettbewerbssteuerung werden verstärkt zum Einsatz kommen: auf die Umsetzung der Prinzipien von Zugänglichkeit, Gleichbehandlung, Erschwinglichkeit sowie die Einhaltung von Qualitätsstandards wird zukünftiglich ebenso Augenmerk gelegt wie darauf, dass Selektion verhindert und Inklusion gefördert wird. Der Wettbewerb muss sich am Gemeinwohl orientieren.

An Programmatik fehlt es der EU auf diesem Gebiet demnach nicht, sodass eine Überprüfung der weiteren Entwicklung von dieser Seite her möglich erscheint. Programmatik ist das eine, Realität das andere. Wie werden die hier skizzierten Prozesse in wissenschaftlichen Publikationen beurteilt?

In wissenschaftlichen Publikationen werden die Schwächen der bisherigen Veränderungsprozesse ebenso deutlicher gesehen wie die Risken der konsequenten Einführung des Wettbewerbsprinzips betont. Inhaltlich betreffen die Kritikpunkte die Form von Privatisierungen, den Mangel an konzeptiver Prägnanz der Programme und den Mangel an unabhängiger Evaluationsforschung.

Diese Überlegungen sind allgemeiner Natur und wurden unabhängig von unserem Beispiel in Baden-Württemberg entwickelt. Insgesamt fällt auf, dass es auf diesem Gebiet des Wandels von Trägerschaftsystemen kaum empirische Untersuchungen gibt, was mit dazu führt, dass die Diskussionen zum Gegenstand merkwürdig diffus geführt werden. Oftmals handelt es bei den Analysen um die bloße Explikation von Meinungen (vgl. Olk 2001).

\section{Privatisierungsversagen}

Entstaatlichung ist ein Trend der Zeit. Dieser Trend nährt sich u.a. aus dem Unbehagen der Menschen über undurchschaubare staatliche Bürokratien und parteipolitischen Einfluss auf ihr Alltagsleben. Rücknahme von Politik und Sachorientierung der Akteure sollen die Effizienz der Sozialsysteme steigern: mit geringerem Einsatz von Steuermitteln soll den wirklich Bedürftigen professionell geholfen werden. Wenn schon nicht recht geglaubt wird, dass sich die Budgetmittel kürzen lassen, so soll doch die progrediente Steigerung gebremst werden. Im politischen Marketing wird gerne beides verknüpft. Das kommt bei den Menschen, die zunehmend unter der Leistungsverdichtung des Arbeits-, Freizeitund Familienlebens leiden, gut an: »Mir hilft niemand, so soll auch niemandem geholfen werden, der es nicht »wirklich« braucht!«

Doch empirisch betrachtet zeigen die zahllosen in den letzten Jahren abgewickelten formellen oder unechten Privatisierungen, das sind Ausgliederungen und Verselbständigungen von ehemaligen staatlichen Dienststellen bei Beibehaltung der Eigentümerstruktur, bisweilen Gegenteiliges (Prisching 2003):

- Sie erhöhen häufig die Zugriffsmöglichkeiten der Politik;

- Ihre leitenden Akteure büßen die ehedem mühsam gegen absolutistische Herrscher erkämpften Rechte des Beamtentums ein;

- Sie senken die für die politischen Instanzen anfallenden Risiken unsachlicher bis korrupter Eingriffe;

- Sie schaffen ein Feld neuer Optionen für politische Gabenverteilung und zuletzt

- Gelingt es ihr (bislang) sich selbst zu legitimieren.

Bei der politischen Idee der Privatisierung handelt es sich um ein ideologisches Konstrukt, die den an der Macht befindlichen politischen Parteien kompensatorisch Einfluss sichert, kompensatorisch insofern, als sie den von ihnen selbst herbeigeredeten Verlust an Macht und Verantwortung durch die Möglichkeit der Einflussnahme im »Kleinen« etwas ausgleicht. Unter den an der Macht befindlichen politischen Parteien ist die Idee der Privatisierung daher konsensfähig. Doch wer zahlt den Preis dafür? Für Dimmel (2005, 58 f) steht fest: Das gegenwärtige System einer formellen Vergabepflicht, einem der Merkmale unechter Privatisierungen, ohne gleichwertige Festlegung von Qualitätskriterien sozialer Dienste führt nach und nach in einen autoritären Sozial- und Wohlfahrtsstaat. Die Macht der (Partei-) Politik steigt wie die Ohnmacht der Leistungserbringer und Klienten.

Im Bereich der Strafvollzugspolitik spricht noch ein weiteres politisches Kalkül für unechte Privatisierungen: Strafvollzugspolitik verfügt über ein großes Skandalisierungspotential. Die Bürger identifizieren sich zugleich mit den vermeintlichen Opfern wie Tätern, ihre Einstellung zu Strafvollzug, Bewährungshilfe, Gerichtshilfe, Außergerichtlichem Tatausgleich usw. ist stets ambivalent und die Stimmung kann schnell umschlagen. Die gefühlsmäßigen Reaktionen auf Ereignisse können sehr heftig sein und der politischen Karriere der politischen Amtsinhaber abträglich. Mehr Distanz zu diesen Einrichtungen im öffentlichen Meinungsbild reduziert erheblich das Risiko des politischen Absturzes.

Es erscheint daher notwendig, neue rechtliche Instrumente der Kontrolle von Privatisierungen zu schaffen, da die klassischen Instrumente der Komplexität der politischen und finanziellen Interaktionen und Transaktionen nicht gewachsen sind. Kontrollierbarkeit im Bereich von unechten Privatisierungen liegt nicht mehr in notwendigem Ausmaß vor, was in keinem Verhältnis zum möglichen Schaden liegt. Hingegen wird eine Reduktion parlamentarischer Einfluss- und Kontrollleistungen beobachtet (Trube $u$. Wohlfahrt 2001, 35).

\section{Mangel an konzeptiver Prägnanz}

Im europäischen Einigungsprozess ergeben sich eine Fülle von Missverständnissen aus der Vielfalt der Sprachen und den komplexen Problemen der Übersetzung und Übertragung von Begriffen. Der Begriff der "Subsidiarität «, der in vielen Ländern ein zentraler Begriff in der Geschichte der Entwicklung der Trägersysteme ist, wird sehr unterschiedlich verstanden, woraus sich zunächst ein Mangel an konzeptiver Prägnanz ergibt. Die Wahrnehmung dieses Problems birgt aber auch die Chance, in Diskursen die bisher verwendeten Begriffe hinsichtlich ihrer latenten Kontexte zu untersuchen und dann neue prägnante Konzepte $\mathrm{zu}$ entwickeln. Was könnte "Markt «, »Ökonomie«, »Wettbewerb « usw. in Zukunft bedeuten? Die Chancen für einen sprachsensiblen Diskurs stehen allerdings schlecht, da verlässt man sich schon eher auf die eingeübten Muster der Sprachkolonialisierung, die nur zwei Ergebnisse zulassen: Unterwerfung oder Scheinkonsens.

Unser Beispiel der Privatisierung demonstrierte bereits das Problem des Mangels konzeptiver Prägnanz. Versuchen wir es an einem weiteren geläufigen Begriff, dem des Wettbewerbs: Ein konstitutives Merkmal des europäischen Einigungsprozesses ist das Bekenntnis zur Sicherstellung des »freien Wettbewerbs«. Dazu werden Regeln für die Finanzierung, Aufsicht, Ausschreibung, Vergabe und Leistungskontrolle aus- und überarbeitet. Ziel ist es, dass Unternehmen angehalten werden, bestmögliche Leistungen $\mathrm{zu}$ erbringen, der Wettbewerb soll die gut Arbeitenden belohnen und die schlecht Arbeitenden bestrafen. Doch, so fragen sich die kritischen Geister: handelt es sich um einen Preiswettbewerb oder 
um einen Qualitätswettbewerb oder eine Mischform der beiden? Was als Begriffsinstrumentarium bei der Herstellung von Produkten ausreichend definiert zu sein scheint, wird im Bereich persönlicher Dienstleistungen als Ko-Produktion schnell unklar:

- was heißt Wettbewerb in diesem Zusammenhang?

- Was sind die Kriterien dieser Wettbewerbe?

- Welche Ziele haben sie, welche Formen, welche Folgen?

Phänomene des Sozialen gehören evidenterweise zu einem anderen Realitätsbereich als Phänomene der Technik (Posch 2005). Sozialarbeiterische Leistungen können nur gewaltsam der Wettbewerbslogik unterworfen werden. Dies hat die EU-Kommission mittlerweile erkannt und in den neuen Diskussionen wird, wie ich oben gezeigt habe, nicht mehr von Wettbewerb, sondern von "gesteuertem Wettbewerb « gesprochen. Was sprachlich eine Einschränkung bedeutet, wirft in der Praxis erhebliche Probleme auf.

Hier zeigt sich eine weitere Sackgasse in der bisherigen Diskussion: die EU ist angetreten, ganz im Sinne der oben erwähnten Tendenz zur Entstaatlichung, zu »de-regulieren«: »Öffnet die Märkte und vertraut den Regeln des Marktes! « klingt himmlisch in den Ohren der Eliten, für die Durchschnittsbürger klingt das heute nicht mehr so gut wie vor 10 Jahren. Regeln schützen die Rechte der Schwachen. De-Regulierung hilft den Eliten ihre Positionen zu stärken. Weiter zeigte sich, dass die Marktöffnung paradoxerweise mit dem Wiedererstarken regulativer Politik einherging. Sowie dies nicht mehr zu übersehen war und die mediale Kritik am Bürokratismus der EUVerwaltung einsetzte, kippte man eine Reihe von EU-rechtlichen Bestimmungen; gar nicht überraschend vor allem im Bereich des Naturschutzes. Darin zeigt sich einerseits eine Konfliktdynamik aber auch ein Stillstand der Entwicklung. Die politischen Eliten (die »symbolischen Arbeiter «) haben einiges an Glaubwürdigkeit eingebüßt und es wird im Zusammenhang unseres Themas die Frage aufgeworfen: Was bedeutet denn die Forderung der politischen Eliten nach mehr Wettbewerb im Bereich persönlicher Dienstleistungen?

Wohlfahrt (2005) kam in seiner Analyse der Folgen von mehr Wettbewerb $\mathrm{zu}$ folgenden Ergebnissen:

- »Mehr Wettbewerb« bedeutet, dass die Sozialunternehmen von Mitgestaltern der Sozialpolitik zu Leistungserbringern mutieren.

- »Mehr Wettbewerb« ersetzt (zumindest in Deutschland) das Subsidiaritätsprinzip;

- »Mehr Wettbewerb« vergrößert Planungsrisiken für Träger wie ihre Beschäftigten.

- Die mit der Forderung nach »mehr Wettbewerb« verbundene Einführung des Kontraktmanagements hat zum Ziel, ein effizient gestal- tetes, besser integriertes Versorgungssystem zu etablieren, jedenfalls hat nun der »aktivierende Staat« ein Instrument effizienter Angebotssteuerung in der Hand. Das kann gut gehen, wenn er entsprechendes Fachpersonal zur Verfügung hat.

- »Mehr Wettbewerb« hat jedenfalls Auswirkungen auf die Organisationsstrukturen der Sozialunternehmen:

- durch Differenzierung des Leistungsangebots wird die Anpassungsfähigkeit erhöht;

- die Strukturen werden hierarchisiert und zentralisiert, um für den Staat als Mittler der Finanzierung berechenbar zu sein;

- »Mehr Wettbewerb «stärkt die Position der Organisationsexperten zu ungunsten der Praxisexperten; er führt zu tarifpolitischer Flexibilisierung, i.d.R. bedeutet dies weniger Einkommen für die Praxisexperten jedoch mehr Einkommen für die Organisationsexperten.

Dementsprechend betrachtet Wohlfahrt die mit mehr Wettbewerb verbundene »Modernisierung « nicht als Fortschrittsentwicklung sondern als »halbierte Modernisierung «. Dies lässt Raum für weiteres Nachdenken und Präzisierung der Konzepte.

\section{Mangel an unabhängiger Evaluations- forschung}

Es vergeht kaum ein Tag an dem nicht eine Aufforderung auf meinem Schreibtisch landet, dieses und jenes zu evaluieren. Mir hängt ehrlich gesagt das Evaluieren zum Halse heraus: es kostet viel Zeit, die Fragebögen sind schlecht, die Ergebnisse sind oft an den Haaren herbeigezogen und werden in Zusammenhänge gebracht, wohin sie nicht gehören. In einem Fachhochschulstudiengang werden sämtliche Lehrveranstaltungen evaluiert, häufig mit unliebsamen Folgen: z. B. Wissenschaftler sehen sich aufgrund von Evaluierungen gezwungen, sich nicht mehr darum bemühen, Studenten über ihre Forschung zu berichten, um sie am Ende des Semesters zu prüfen, sondern die mutieren zu Pausenclowns, um gute Evaluationsergebnisse zu erzielen. Davon halte ich gar nichts. Evaluieren wurde zu einer schlechten Mode.

Evaluationsforschung ist etwas anderes, sie wird mit sozialwissenschaftlichen Methoden vollzogen und erfolgt nach Prinzipien der Objektivität, Reflexivität und Unabhängigkeit, um die wichtigsten zu nennen. Evaluationsforschung geht davon aus, dass es sich bei Privatisierungen um komplexe, nicht-triviale Prozesse handelt. Sie ist daher aufwändig und kostspielig. Das Projekt der Transformation der Bewährungs- und Gerichtshilfe in Baden-Württemberg sollte ursprünglich vom Institut für Kriminologie Tübingen evaluiert werden, die Evaluationsforschung musste aber mangels Finanzierungsmittel zurückgestellt werden, was auch Dr. Steindorfer bedauert. Bei einem Projekt dieser Größenordnung und Tragweite wäre es jedoch notwendig und sinnvoll, mit dem zweiten Abschnitt auch die Evaluationsforschung zu starten.

\section{Zwischenresümee}

Der europäische Integrationsprozess bietet auch auf dem Gebiet der Internationalisierung von Trägerschaften personenbezogener Dienstleistungen gute Chancen, aber birgt auch einige Risiken in sich. Chancen wären wahrzunehmen, Risiken ernst zu nehmen. Dies ist nur in reflexiven Prozessen möglich, an denen neben den Vertretern der Ministerien und Trägern auch deren MitarbeiterInnen und nicht zuletzt die KlientInnen partizipativ beteiligt sein sollen.

Für den traditionsreichen Verein »Neustart« bietet die Ausdehnung seiner Tätigkeiten von Österreich nach Deutschland auch Chancen für die Entwicklung in Österreich. Dazu ein Beispiel: war noch vor vier Jahren die Weiterführung der ehrenamtlichen Bewährungshilfe Gegenstand heftiger Diskussionen, zeigt sich nunmehr, dass diese zur hauptberuflich durchgeführten komplementäre Praxis der Bewährungshilfe ein international geschätztes Konzept darstellt. Es ist ein Beispiel gelungener Differenzierung des Leistungsangebots und steht nun nicht mehr zur Diskussion.

Will die Internationalisierung von Trägerschaften erfolgreich sein, setzt dies u . a. voraus, dass lokale Traditionen ernst genommen und geprüft werden. Dies bietet auch die Möglichkeit, schlechte Traditionen als solche zu erkennen und abzustellen. Auch das wäre als Innovation zu verstehen. Ein wichtiges Markenzeichen der österreichischen Bewährungshilfe, wonach hauptberuflich tätige Bewährungshelfer nicht mehr als 30 Probanden betreuen dürfen, könnte sich auf diese Weise nach und nach auch europaweit durchsetzen. Das würde nach den Erfahrungen in Österreich zwar die Kosten für die Bewährungshilfe etwas erhöhen, der Nutzen der so gestalteten Bewährungshilfe würde jedoch in weit höherem Ausmaß gesteigert werden. Europa muss in intelligente Maßnahmen investieren, um Gesamtkosten zu sparen. Intelligente Maßnahmen sollen zwischen Politikern, Beamten der Ministerien, Managern der Träger, Praktikern, Klienten und Wissenschaftlern in fachpolitischen Diskursen entwickelt werden. Dabei sind neue Kompetenzen gefragt (Herrenbrück 2005).

Nicht zuletzt zeigt dieses Beispiel, dass der Mut, die altbewährten Pfade des Korporatismus zu verlassen, sich auszahlt und es ergibt sich daraus die Anregung, dass andere Justizministerien wie auch das Österreichische Justizministerium dem Beispiel des Baden-Württembergischen folgen und in angemessener Frist und nach entsprechenden konzeptiven Vorarbeiten die Durchführung der Aufgaben der Bewährungshilfe ausschreiben. Wettbewerb kann keine Einbahnstraße bleiben. 
Die mit den europäischen Trends bei der Weiterentwicklung der sozialen Sicherungssysteme aufgezeigten Probleme sind schwerwiegend, jedoch grundsätzlich wenn schon nicht lösbar, so doch meisterbar. Perfekte Lösungen liegen in weiter Ferne: Am Himmel sehen wir einige wenige Schwalben, die jedoch noch nicht den Sommer bringen werden.

Der Verfasser ist Studiengangsleiter "Sozialarbeit" an der FH JOANNEUM Graz.

\section{Literatur}

Dimmel, N. (2005): Zur Anwendung des Vergaberechts auf die Auftragserteilung und Vertragsgestaltung sozialer Dienste in Österreich. In: ders. (Hg.): Perspektiven der Sozialwirtschaft 2005 - 2015. Wien 2005 (LIT-Verlag) S. 17-66

Evers, A. (2005): Zum Verhältnis von Staat und Sozialwirtschaft im Bereich sozialer Dienstleistungen. Eine Auseinadersetzung mit den Konzepten der Kommission der Europäischen Gemeinschaften. In: Dimmel, N. (Hg.): Perspektiven der Sozialwirtschaft 2005 -2015. Wien 2005 (LIT-Verlag), S. 100 - 126

Herrenbrück, S.(2005): Neue Kompetenzen gefragt. In: Sozialwirtschaft 6, S. $6 \mathrm{f}$

Olk, Th. (2001): Träger der Sozialen Arbeit. In: Otto, H.-U. u. H. Thiersch: Handbuch Sozialarbeit, Sozialpädagogik. Neuwied 2001 (Luchterhand) S. 10910-1926

Posch, K.(2005): Beziehungs- und lebensweltorientierte Soziale Arbeit. Praktische, theoretische und organisatorische Aspekte. In: sozialextra 6, S. 35-39

Prisching, M. (2003): Entstaatlichung als Politisierung. In: Novak,M.: Res Universitatis.Bernd-Christian Funk zum 60. Geburtstag. Wien, Graz (Neuer wissenschaftlicher Verlag) S. 203-229

Trube / Wohlfahrt / Boeßenecker Hrsg. (2004): Privatisierung im Sozialsektor

Wohlfahrt, N. (2005): Zur Transformation der Sozialwirtschaft. Strategische Unternehmensentwicklung in der Sozialwirtschaft zwischen Kontraktmanagement, Wettbewerbsrecht und sozialpolitischen Selbstverständnis. In: Dimmel, N. (Hg.): Perspektiven der Sozialwirtschaft 2005-2015. Wien 2005 (LIT-Verlag), S. 67-99

www.eupik.org Newsletter vom 6.10.2005

www.soziale-dienste-ineuropa.de

\section{Satzfehler in der NK im Jahre 2005}

Beginnen wir mit Heft 2-2005.

Der Nachruf zum Tode von Helga Einsele stammt von Helga Maelicke und nicht - wie versehentlich auf dem Deckblatt steht: Madicke.

Fahren wir fort mit Heft 4-2005.

Leider sind Deckblatt und Inhaltsverzeichnis völlig missverständlich. Auf dem Deckblatt dieses Heftes fehlt Hans Theile. Im Inhaltsverzeichnis und im Text erscheinen stattdessen zu Unrecht zwei Beiträge von Eduard Güroff. Dieser Autor hat den ersten Titelbeitrag geschrieben: Die Staatsanwaltschaft im Wirtschaftsstrafverfahren. Der zweite Titelbeitrag stammt hingegen von Hans Theile. Wir bitten dies zu entschuldigen.

Sie finden unter Zeitschriften auf der Webseite des Verlages www.nomos.de einen korrekten Ausdruck als Sonderdruck mit dem Titel: Wirtschaftsstrafverfahren: Entstehung eines neuartigen Verfahrenstyps, von Hans Theile, NK 42005, S. $142-146$.

Sie können diesen Sonderdruck auch auf meiner Webseite herunterladen: www.uni-kiel.de/isk/cgi-bin/NK.html

Monika Frommel

\section{PERSONALIA}

\section{Bernd Maelicke, neuer Vorsitzender BAG-S}

Am 13. Dezember 2005 wurde der Mitherausgeber dieser Zeitschrift Prof. Dr. Bernd Maelicke durch die Mitgliederversammlung der Bundesarbeitsgemeinschaft für Straffälligenhilfe ( BAG-S ) zum neuen Vorsitzenden gewählt. Seit den 60er Jahren ist er ehrenund hauptamtlich in der Straffälligenhilfe tätig. Von 1978 bis 1990 war er Direktor des Instituts für Sozialarbeit und Sozialpädagogik ( ISS ) in Frankfurt a. M., von 1990 bis 2005 Ministerialdirigent im Ministerium für Justiz, Europa und Soziales in Kiel. Seit Mai 2005 ist er Professor für Sozialmanagement und Devianzmanagement an der Universität Lüneburg.
Er ist angetreten, um die BAG-S in kürzester Zeit zu sanieren und für sie klare Zukunftsperspektiven zu entwickeln und abzusichern. Dazu bedarf es der Unterstützung aller Mitglieder - diese wurde auf der Mitgliederversammlung einhellig zugesichert. Zunächst geht es darum, das aktuelle Defizit abzubauen, um so wieder die Voraussetzungen für die Bundesförderung zu sichern. In einem zweiten Schritt sind dann effektive und effiziente Organisationsstrukturen zu entwickeln - alles mit dem Ziel der Stärkung der gesamten Straffälligenhilfe auf Bundes-, Landes- und kommunaler Ebene. 